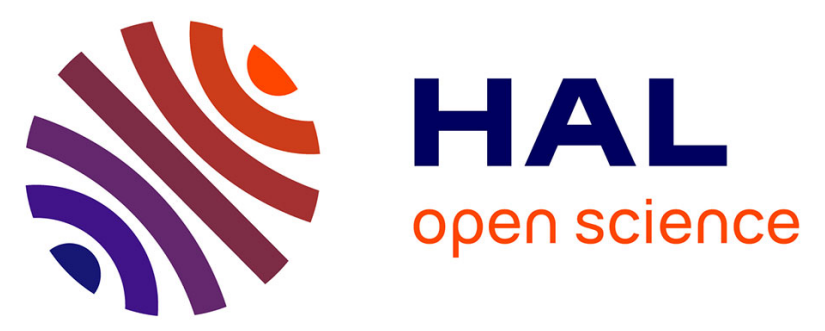

\title{
Boosting the analysis of protein interfaces with Multiple Interface String Alignments: illustration on the spikes of coronaviruses
}

Stéphane Bereux, Bernard Delmas, Frédéric Cazals

\section{- To cite this version:}

Stéphane Bereux, Bernard Delmas, Frédéric Cazals. Boosting the analysis of protein interfaces with Multiple Interface String Alignments: illustration on the spikes of coronaviruses. Proteins - Structure, Function and Bioinformatics, 2022, 90 (3), pp.848-857. 10.1002/prot.26279 . hal-03387889

\section{HAL Id: hal-03387889 \\ https://hal.inria.fr/hal-03387889}

Submitted on 20 Oct 2021

HAL is a multi-disciplinary open access archive for the deposit and dissemination of scientific research documents, whether they are published or not. The documents may come from teaching and research institutions in France or abroad, or from public or private research centers.
L'archive ouverte pluridisciplinaire HAL, est destinée au dépôt et à la diffusion de documents scientifiques de niveau recherche, publiés ou non, émanant des établissements d'enseignement et de recherche français ou étrangers, des laboratoires publics ou privés. 


\title{
Boosting the analysis of protein interfaces with Multiple Interface String Alignments: illustration on the spikes of coronaviruses
}

\author{
S. Bereux*, B. Delmas $\stackrel{\text { F }}{\text { F. Cazals }} \ddagger$
}

October 20, 2021

Keywords: protein interface, multiple sequence alignments, interface comparison, interface RMSD, Structural Bioinformatics Library, coronaviruses, spikes, fusion proteins.

Running title: Multiple Interface String Alignments

*Université Côte d'Azur, Inria, France, École polytechnique

${ }^{\dagger}$ INRAe

‡Université Côte d’Azur, Inria, France. Correspondence: Frederic.Cazals@inria.fr 


\begin{abstract}
We introduce Multiple Interface String Alignment (MISA), a visualization tool to display coherently various sequence and structure based statistics at protein-protein interfaces (SSE elements, buried surface area, $\triangle \mathrm{ASA}, \mathrm{B}$ factor values, etc). The amino-acids supporting these annotations are obtained from Voronoi interface models. The benefit of MISA is to collate annotated sequences of (homologous) chains found in different biological contexts i.e. bound with different partners or unbound. The aggregated views MISA/SSE, MISA/BSA, MISA/DASA etc make it trivial to identify commonalities and differences between chains, to infer key interface residues, and to understand where conformational changes occur upon binding. As such, they should prove of key relevance for knowledge based annotations of protein databases such as the Protein Data Bank.

Illustrations are provided on the receptor binding domain (RBD) of coronaviruses, in complex with their cognate partner or (neutralizing) antibodies. MISA computed with a minimal number of structures complement and enrich findings previously reported.

The corresponding package is available from the Structural Bioinformatics Library (http://sbl. inria.fr

and https://sbl.inria.fr/doc/Multiple_interface_string_alignment-user-manual.html).
\end{abstract}




\section{Introduction}

Protein complexes and their interface. Understanding the stability and the specificity of protein interactions is a fundamental problem [1, both to unveil complex biological mechanisms and design therapeutics. Quantitative insights on such problems call for analysis falling in the realm of thermodynamics and kinetics. On the thermodynamics side, the strength of an interaction is given by the binding affinity, measured say by the dissociation constant $K_{d}[2$. The affinity is a subtle thermodynamic quantity, encompassing an enthalpic term derived from the interaction potential energy, and an entropic term related to the entropic penalty upon binding - see e.g. 3, 4. From the theoretical standpoint, the binding affinity can be defined as the ratio of three so-called partition functions [5]. These partition functions are themselves defined from the potential energy of the system, which in particular encodes interaction energies at play between atoms (electrostatic interactions, non covalent interactions, bonded interactions). This observation does not settle the question though, as the huge dimensionality of the system involving the biomolecules and the solvent precludes calculations, except maybe for small systems using massive calculations [6, 7]. Application-wise, an illustrative example of the shear difficulty of obtaining accurate affinity estimates is provided by the affinity maturation of antibodies in the immune response [8]. As illustrated by this case study, the same (backbone) bound geometry may indeed corresponding to dissociation constants differing by two orders of magnitude. On the kinetics side, the residence time (time spent by a ligand in contact with its biological target) is $1 / K_{\text {off }}$ 9, 10]. Estimating reaction rates $\left(K_{\mathrm{on}}\right.$ and $\left.K_{\mathrm{off}}\right)$ is also extremely difficult.

This state of affairs partly explains the success of models based on geometric proxys (distances, surfaces and volumes) to estimate structural, thermodynamic, and kinetic properties. Such models originate with the seminal work of Richards for individual molecules [11] and Janin for complexes [12. They proved instrumental to understand the specificity and affinity of interactions [1]; in some cases, they even proved good enough to estimate binding energies up to (of the order of) $1.4 \mathrm{kcal} / \mathrm{mol}$ [13]. To study two partners forming a complex, such models require identifying the atoms in contact. To this end, Voronoi models, be their affine [14] or curved [15, provide a parameter free approach to model interfaces, and affine models in particular give a direct access to various structural parameters including the number of interface atoms and residues, the buried surface area, the number of binding patches, or the interface curvature [14].

These parameters are typically sufficient to compare interfaces for cases belonging to various protein families (protease-inhibitors, enzyme-substrate, antibody-antigen, signal transduction, etc) [16], or cases within a protein family [17. On the other hand, in processing complexes specifically addressing a biological case, say a family of homologous proteins targeting the same cognate partner, it is also critical to study correlations between structural parameters describing interfaces and sequence related pieces of information. For sequences, the natural route in to resort to multiple sequence alignments (MSA) [18].

Multiple Interface String Alignment (MISA). In this work, we introduce MISAs, a visualization tool to display coherently various sequence and structure based statistics at protein-protein interfaces (SSE elements, buried surface area, $\triangle \mathrm{ASA}, \mathrm{B}$ factor values, etc). The benefit of MISAs is to collate annotated sequences of (homologous) chains found in different biological contexts (unbound or bound with different partners).

We illustrate the interest to analyse the receptor-binding domain (RBD) or the virus SARS-Cov-2 causing the Covid19 outbreak. First, we compare the receptor-binding domains (RBD) of SARS-Cov-1 and SARSCov-2 (two phylogenetically-related betacoronavirus). Second, we compare the interfaces of their RBD bound to their common cell receptor and with antibodies.

The implementation of MISAs available in the SBL (http://sbl.inria.fr, [19]), see https://sbl. inria.fr/doc/Multiple_interface_string_alignment-user-manual.html. 


\section{Methods: MISAs - multiple interface string alignments}

\section{$2.1 \quad$ MISA}

We assume a collection of crystal structures of the bound and unbound types. We call a polypeptide chain in these structures a chain instance. We also assume that a multiple sequence alignment is available for the sequences of the instances of interest. Practically, we expect two settings: (i) chain instances with identical sequence: since numberings used in the respective PDB files are identical, the identity alignment is used; (ii) homologous proteins: a multiple sequence alignment providing a coherent numbering of amino acids (a.a.) is taken for granted.

The terminology used in the sequel is as follows. We study chain instances and compare them against one another using one MISA for each so-called MISA id. A MISA is based on interface strings (i-string), which following conventions for sequence alignments, use the one-letter code of (a.a.) plus three additional symbols $\left\{z_{-} *\right\}$. We now detail these concepts.

MISA id. A complex $C_{i}$ is specified by two sorted lists of chains ids i.e. $C_{i}=\left(\left\{A_{j}\right\},\left\{B_{j}\right\}\right)$ for the two partners; each list is endowed with a partner/structure name. Likewise, an unbound structure is specified by a sorted list of chain ids $U_{i}=\left\{A_{j}\right\}$, and is also given a partner/structure name (e.g. antibody, antigen, etc.) We consider a collection of complexes $\mathcal{C}=\left\{C_{i}\right\}$, and optionally a collection of unbound structures $\mathcal{U}=\left\{U_{i}\right\}$. The minimal setup is naturally that of a single complex without any unbound structure.

Our goal is to build one MISA for each so-called MISA id, which we formally define as:

Definition. 1 The MISA id of a chain in a complex or unbound structure is the string defined by the structure name followed by the index of the chain in its sorted list $\left\{A_{j}\right\}$ or $\left\{B_{j}\right\}$. (Nb: by convention, indices start at 0.)

Note that because of the sortedness, a given chain gets the same MISA id in a complex or unbound structure.

Example 1 Consider two antibody-antigen complexes. $C_{1}=(\{H, L\},\{A\}), C_{2}=(\{M, N\},\{B\})$, each involving the heavy chains (chains $H$ and $M$ ), the light chains (chains $L$ and $N$ ), and the antigen (chains $A$ and B). The two structure names are thus antibody (IG for short) and antigen (Ag for short). The MISA id of the heavy chain is $I G_{-} 0$, that of the light chain $I G_{-} 1$, and that of the antigen $\mathrm{Ag}_{-} 0$.

Voronoi interface in a binary complex. The two lists of chains in the specification of a complex $C_{i}$ define the so-called partners in the complex, called $A$ and $B$ for short. In the sequel, we build the Voronoi interface for these partners, using the Voronoi (power) diagram of the solvent accessible model [14, 1, 20, More precisely, recall that the Voronoi model identifies pairs of atoms, one on each partner, which are either directly in contact as their Voronoi cells are neighbor, or are contacting a common crystallographic water molecule. Therefore, an a.a. contributing at least one interface atom is called an interface residue/a.a..

Practically, we use the sbl-intervor-ABW-atomic.exe executable from the package Space_filling_model of the Structural Bioinformatics Library ([19], http://sbl.inria.fr).

Interface strings (i-strings). In the sequel, we present MISAs informally, referring the reader to SI Section Supplemental: formal specification of the method. We represent each chain instance with a so-called interface string encoding properties of its interface amino acids. The interface string of a chain instance is a character string with one character per residue, and is actually defined from all instances with the same MISA id. To build this character string, we first build a consensus interface based on all Voronoi interfaces of all complexes in the set $\mathcal{C}$. At each position of the consensus interface, the most frequent residue observed in all the bound structures is chosen as the consensus residue-with ties broken using the alphabetical order of the one letter code of a.a.. Using this consensus interface, the residues of a given chain instance (bound or unbound) are assembled into the so-called interface string: 
Definition. 2 The interface string (i-string) of a chain instance is the string with one character per amino acid, defined as follows:

1. Residue not part of the consensus interface:

- Displayed with a dash "-" if it is part of the crystal structure, and underscore " _ " otherwise.

2. Residue part of the consensus interface:

- Residue not found in the crystal structure: displayed with the star '*,.

- Residue found at the interface for this particular chain: displayed with the uppercase one letter code if the a.a. matches the consensus a.a., or with the lowercase letter otherwise.

- Residue not found at the interface for this particular chain, even though the corresponding position contributes to the consensus interface (for other chains): displayed in an italicized uppercase/lowercase letter. (Note that this is the case for all residues of unbound structures-as no partner implies no interface.)

Practically, it is often sound to restrict the study to a range of amino-acids on the chains studied, whence the following:

Definition. $3 A$ window series for a MISA id is a sequence of integer ranges of residue ids $\left\{\left(B_{i}, E_{i}\right)\right\}$ used to restrict the display of the $i$-string.

MISA. We use the MSA to align the symbols of interface strings, yielding:

Definition. 4 The MISA of all chain instances in $\mathcal{C} \cup \mathcal{U}$ with the same MISA id is the multiple alignment of their interface strings.

\subsection{Colored MISAs}

We further color the letters of a MISA to encode properties of biological/biophysical interest.

MISA/SSE: coloring based on Secondary Structure Elements. The type of SSE a given a.a. belongs to is especially useful when comparing bound and unbound structures, to assess perturbations in the hydrogen bonding network. Practically, we use the dictionary of SSE from [21].

MISA/BSA: coloring based on Buried Surface Area. Consider a chain instance in a complex. The BSA of this chain is defined as the accessible surface area (ASA) [11] of this chain in the partner alone minus the ASA of the chain in the complex. We report the BSA on a per residue basis, computed using the algorithm from [22].

Remark 1 The Voronoi interface model identifies privileged contacts between atoms of the partners. Interestingly, selected interface atoms, generally backbone atoms, can be buried within their own subunit [23]. Since such atoms do not have any BSA (they do not have ASA in their own subunit), a residue containing only such atoms is an interface residue for which the BSA information is irrelevant.

MISA/D ASA: coloring based on the variation of accessible surface area. A limitation of the BSA is that its calculation uses the geometry of the bound structure only. The calculation is thus oblivious to conformational changes which may be at play in case of induced fit or conformer selection. To mitigate the previous plot, we also provide the so-called $\Delta$ ASAcoloring scheme.

Consider an interface partner $P(=A$ or $B)$, in the complex, and consider the i-th residue of one of its chains. Let $A S A_{i}^{b}$ be the ASA of this i-th residue in the structure involving only the chains defining partner $P$. Also denote $\overline{A S A}_{i}^{u}$ the average ASA of the i-th residue in unbound structures with the same MISA id. We compute for the i-th residue of a bound structure the quantity $A S A_{i}^{b}-\overline{A S A}_{i}^{u}$ and display it with a color map. 
Remark 2 The quantity $A S A_{i}^{b}-\overline{A S A}_{i}^{u}$ is undefined when the corresponding residues are absent in the unbound crystal structures (* in i-string).

MISA/B-factor: coloring based on B-factors. The B-factors reflects the atomic thermal motions. Selected recent crystal structures report this information as a $3 \times 3$ ANISOU matrix (the anisotropic Bfactor). In order to have a single quantity for all the crystals, the ANISOU matrix $B_{\text {anisou }}$ is converted into B-factor thanks to the formula $B_{\text {factor }}=\operatorname{trace}\left(B_{\text {anisou }}\right) / 3$ [24]. Optionally, one can choose to normalize the B-factor with respect to (i) all the residues in the chain, or (ii) all the displayed residues.

\subsection{Availability and practical matters}

MISAs are implemented within the Multiple_interface_string_alignment package of the Structural Bioinformatics Library ([19, http://sbl.inria.fr), see https://sbl.inria.fr/doc/Multiple_interface_string_alignment-user-manual.html.

The calculation and the analysis of MISAs is based on four scripts-see online documentation:

- sbl-misa.py: building MISAs from a description of complexes and possibly unbound structures.

- sbl-misa-mix.py: mixing selected colored MISAs into a single (html) file.

- sbl-misa-bsa.py: displaying the BSA of all (or selected user defined) residues.

- sbl-misa-diff .py: comparing i-strings and associated properties (in particular BSA) of two interfaces. Has two modes: comparing two i-strings; comparing one i-string and a list of interface residues typically coming from a publication.

Illustration of these scripts to investigate the questions discussed in the next section are provided in the jupyter notebook which accompanies our software.

\section{Results: studying the receptor-binding domain (RBD) of coron- aviruses}

Similarly to other enveloped viruses, the SARS-Cov-2 virus enters cells using fusion proteins. Having recalled the known facts on this mechanism, we show that MISAs are helpful in two respects: (i) to automate such studies and refine some of the findings reported so far, and (ii) to provide a coherent view of several sequence-structure based statistics, which is instrumental in particular to understand the binding affinity of SARS-Cov-2 RBD for its target.

\subsection{Biological problem}

Coronavirus entry into cells is mediated by the so-called spike that is responsible for receptor binding and membrane fusion [25, 26]. The covid19 outbreak triggered an impressive number of structural studies on the entry of SARS-Cov-2, namely [27 [28 [29] [30 [31 [32] 33]. Interactions between the spikes of SARS-Cov-2 with antibodies have also studied [34, [35, [36]. These papers naturally unveiled critical aspects of this complex process, based on experiments and also sequence based and structure based analysis.

Spikes are homotrimers of the protein S [37, 38]. Each chain consists of two domains S1 and S2, separated by cleavage sites denoted S1/2 and S2' [39, 33, 35]. Domain S1 contains the receptor-binding domain (RBD), while domain S2 contains the machinery responsible for the fusion of the virus envelope with the membrane of the cell. In short, the main steps of the mechanism are as follows 39, 29: (1) Attachment of the RBD to its target thanks to the receptor binding-motif (RBM), (2) proteolysis cleavage/activation at S1/S2 removing the S1 subunit, (3) second cleavage at S2', refolding of fusion machinery - anchoring of fusion peptide into the target membrane, (4) membrane fusion and genome delivery into the cytoplasm. In human, 
both SARS-Cov-1 and SARS-Cov-2 targets ACE2 [32], a membrane bound enzyme catalysing the hydrolysis of angiotensin II into angiotensin, as a receptor to enter into cells. The following facts are noticeable [29]. The virulence of SARS-Cov-2 owes in particular to the presence of a polybasic cleavage site (insertion of three R) collated to the usual S1/S2 cleavage site. The RBD of SARS-Cov-2 stands up less often than that of SARS-Cov-1, which likely favors evasion from the immune system. (Nb: the two conformations are called up/down or closed/open.) This also disfavors binding to the target (binding in the down configuration yields steric clashes), but the lesser proportion of RBD standing up is rescued by a higher binding affinity for ACE2 [29]. Interestingly, a phylogenetically distant human alphacoronavirus, HCoV-NL63 also targets ACE2 as a receptor.

In the following, we illustrate the ability of interface string and MISAs to shed light on these complex facts. In doing so, our goal is a presentation of the merits of MISAs, rather than an arbitration of the merits of different publications which investigated the same systems. For this reason, we use a minimal number of crystal structures to get informative MISAs, namely two bound and two unbound structures, choosing those with the highest resolution. As we shall see, even such a simple study sheds new light on a number of published facts.

\subsection{Comparing the RBD of SARS-Cov-1 and SARS-Cov-2 in complex with ACE2}

Baseline. The spikes of SARS-Cov-1 and SARS-Cov-2 are highly homologous proteins binding ACE2. Pairwise alignment shows that the overall percent protein sequence identity is about $76 \%$ [40. The S1 subunit is less conserved (64\% identity) than S2 (90\% identity). Within S1, the receptor-binding domain (RBD) is $74 \%$ identical. The initial analysis of the complex between the RBM of SARS-Cov-1 and its target ACE2 was first carried out in [41]. In this work, it is noted that the BSA at the interface is $\sim 1700 \AA^{2}$, and that the interface involves 18 a.a. on the receptor side and 14 a.a. on the spike side. These analysis are extended to SARS-Cov-2 and other SARS viruses, in particular in [31, SI Fig 1], [30, Fig 2B], and [33, Fig $2 \mathrm{C}$. These recent publications take the 14 a.a. identified in [41] for granted.

Note that in furthering these analysis, we sometimes refer to the jupyter notebook which accompanies our software, and propose selected analysis using the four scripts of the Multiple_interface_string_alignment package.

Whole Voronoi interfaces. The Voronoi interface calculation yields comparable results (Figs. 1 and 2 ). For SARS-Cov-1 (PDBid: 2ajf, RBD: chain E, ACE2: chain A), one obtains 246 interface atoms making up 2 binding patches, and defining overall 414 pairwise contacts. For SARS-Cov-2 (PDBid 6m0j, RBD: chain E, ACE2: chain A), one gets 212 interface atoms also disposed on two binding patches, and defining overall 371 pairwise contacts. The two interface patches correspond to the transition between a beta sheet and loops on the RBD (Fig. 1 (B,C)), dotted line-segments). Moving to interface strings (Fig. 2, we note that the RBD contribution is is characterized by five main stretches along the backbone. Interestingly, two stretches (yellow and red) span the whole interface, crossing on top of one another in a X like figure (Fig. 1. (C)).

Buried surface area. The BSA is essentially proportional to the number of interface atoms [1], and is also a key parameter to estimate binding affinities [42, 13].

We first inspect BSA values obtained for two complexes in each case (Fig. \$1 and Fig. \$2), and compare them against the aforementioned $1700 \AA^{2}$ : SARS-Cov-1: $B S A(2$ ajf chains $\mathrm{E}+\mathrm{A})=925+888=1813 \AA^{2}$; $\mathrm{BSA}(2 \mathrm{ajf}$, chains $\mathrm{F}+\mathrm{B})=864+817=1681 \AA^{2}$; SARS-Cov-2: $\mathrm{BSA}(6 \mathrm{m0j}$ chains $\mathrm{E}+\mathrm{A})=887+843=1730 \AA^{2}$; $\operatorname{BSA}(6 \mathrm{lzg}$, chains $\left.\mathrm{B}+\mathrm{A})=1120+1098=2218 \AA^{2}\right)$. While the values are coherent for SARS-Cov-1, it appears that the complex found in 6lzg features 30\% more BSA. Inspecting the number of interface atoms readily provides the explanation (output of sbl-intervor-ABW-atomic.exe, see jupyter notebook): for 6lzg, one get 156 interface atoms for the RBD, 164 interface atoms for ACE2, and 22 water molecules sandwiched between the partners; these numbers drop to 101,110 and 1 respectively for $6 \mathrm{~m} 0 \mathrm{j}$. This reminds us a well 
known fact, namely that solvation by water molecules with low temperature factors ( 80 units in our case) may significantly change the interface model [14].

Beyond these global values, MISA/BSA gather BSA values for all atoms of the same a.a. (Fig. S1).

Two interesting facts emerge. First, both for SARS-Cov-1 and SARS-Cov-2, the residues which bury the largest ASA are found at both endpoints of the fifth stretch (Fig. S1), contributing to the stabilization of the interface. For SARS-Cov-1, one notes in particular: Y475, T486, Y491; and for SARS-Cov-2: F486, Y489, T500, Y505. We also note that some of these a.a. bury a great deal of SAS (Fig. S2), in particular Y475 for SARS-Cov-1, and Y489 for SARS-Cov-2.

Interface composition. We proceed with the inspection of the number of interface a.a., using the 14 a.a. reported in [41] as baseline.

Focusing on the RBD of SARS-Cov-1, we note that this number is precisely 29 for the crystal structures used (Fig. 2, MISA/SSE). It should be stressed that the this is not an artifact since two atoms are in contact in a Voronoi model provided that their Voronoi restrictions intersect. (Nb: the restriction of a ball is the intersection of the atomic ball and the Voronoi/power region.) We note in particular that three residues omitted from [41] have BSA larger than $30 \AA^{2}$ in (2ajf, chain E): L443: $34.93 \AA^{2}$, P462: $49.79 \AA^{2}$, and I489 : $32.87 \AA^{2}$ (jupyter notebook).

Switching to SARS-Cov-2, these numbers are 27 and 39 respectively, for the aforementioned two crystal structures-a discrepancy owing to solvation by structural water. Letting these count alone, a merit of MISAs appears immediately: as opposed to previous presentations, which single out the 14 a.a. of interest in the whole RBM sequence, it appears immediately that the interface a.a. are located within five main stretches along the sequence.

Interface strings in MISAs also single out the relatively high abundance of tyrosine (Y), and glycine $(\mathrm{G})$ amongst interacting residues. Such a.a. have been shown to cooperatively account for specific recognition in particular in antibody-antigen interactions [43. The role of tyrosines at interfaces is well documented [44, both in terms on interaction types (non polar, cation-pi and $\mathrm{H}$ bonds) and dynamics (the rigid side chain entails a lesser entropic penalty upon binding). Again, this is not visible from previous representations 31 , SI Fig 1], [30, Fig 2B], and [33, Fig 2C] since the interface amino acids are not identified in a systematic manner.

Conformational changes and binding affinity. MISA/SSE show that the RBD of SARS-Cov-2 is much less structured than that of SARS-Cov-1: while all residues are resolved in the unbound structures of SARS-Cov-1, a total of 12 of them are not so in the unbound structures of SARS-Cov-2 processed here (* in the i-strings, Fig. 22. The a.a. concerned are nonpolar (AGGF) or uncharged polar (NSY). Hydrophobic patches on protein surfaces are notorious and contribute to the recognition of cognate substrates [45]. The balance observed here hints at a rather hydrophobic patch also preserving the ability to make hydrogen bonds via NSY. The color changes in MISA/SSE convey the same information: more frequent color changes for SARS-Cov-2 show that binding triggers more significant changes of hydrogen bonding patterns.

The analysis of MISA/ $\triangle$ ASAcomplements this view (Fig. S22). For SARS-Cov-1, the interface a.a. have a balanced behavior, some gaining some loosing ASA, in the mild range $\left(-10 . .+30 \AA^{2}\right)$. For SARS-Cov- 2 on the other hand, the scale is shifted towards negative values $\left(<-150 . .+35 \AA^{2}\right)$. In other words, the binding entails a compactification of the RBD in SARS-Cov-2.

Speaking of conformational changes, we further our analysis with a clustering of structures in terms of IRMSD, which is of special interest to assess the conformational changes between the up and down conformation of the RBD. In this case, no significant difference is observed in terms of IRMSD computed from all backbone atoms (Fig. S3). Phrased differently, MISAs show subtle details at the individual a.a. level, which are overlooked by global IRMSD calculation.

Concluding, the previous discussion shed lights on the determinants of the affinity of the RBDs of SARSCov-1 and SARS-Cov-2 for their receptors [29. For SARS-Cov-2, the unstructured nature hints at a large entropic penalty upon binding. This penalty is clearly counterbalanced by a strong enthalpic component in the dissociation free energy, which we see in particular from the BSA. 


\subsection{Interactions between the RBD of SARS-Cov-2 and antibodies}

Neutralizing antibodies targeting the spikes of viruses can implement various strategies [46, including (i) masking the RBD, (ii) preventing the conformational changes triggering the fusion envelope-membrane, or may be more surprisingly by (iii) mimicking the receptor and triggering early conformational changes of the fusion machinery far away from the cell membrane [34]. We illustrate again the interest of MISAs in this context.

We first consider the complex (SARS-Cov-2-RBD, IG CR3022), the latter being an IG isolated from a convalescent SARS patient. Since this IG also targets the RBD, two critical questions are investigated in [28]: first, the comparison of the complexes complexes SARS-Cov-1-RBD with CR3022 and SARS-Cov-2-RBD with CR3022, so as to understand in particular the lesser binding affinity of CR3022 for SARS-Cov-2- RBD $(\mathrm{Kd}=115 \mathrm{nM}$ versus $\mathrm{Kd}=1 \mathrm{nM})$; second, the absence of in vitro neutralization of SARS-Cov-2 by CR3022, taking into in particular the up/down conformations of the RBD and the role of glycosylation.

Interestingly, the first point use MISAs prepared by hand [28, Fig. 2A]. The systematic nature our automatically generated MISA/SSE shows modest albeit interesting refinements. First, one sees immediately that the regions of the RBD targeted by ACE2 and CR3022 are disjoint (Figs. 3 and 4 jupyter notebook). Second, using the two complexes RBD-CR3022 in the asymmetric unit of the crystal, we note that the interface involves 33 rather than 28 a.a. which bury surface area. Those omitted from [28, Fig. 2A] are sitting on the boundary of the interface though (Fig. 3(B, VdW balls)). MISA/BSA and MISA/ $\Delta$ ASAalso immediately identify those a.a. with the largest contribution to BSA (Fig. 54 and Fig. 95 p. Interestingly too, we note that binding with CR3022 triggers significant conformational changes with significant loss (Y380) or gain (H519) of ASA, as evidence by MISA/ $\Delta$ ASA.

We also compare the interface (SARS-Cov-2-RBD, ACE2) against that of (SARS-Cov-2-RBD, P2B-2F6) [36]. With 10 shared residues, 10 exclusive residues for RBD - P2B-2F6, and 29 exclusive residues for RBD - ACE2, this comparison shows a clear competition between the two binders (jupyter notebook). A direct comparison against the 12 residues reported in [36] also shows that 8 residues reported by the Voronoi model have been omitted (Fig. 3 (C, VdW balls)). Of particular interest are R346 (BSA: 18.18 $\AA^{2}$ ) and T470 (BSA: $\left.21.02 \AA^{2}\right)$.

To complement this analysis, we consider the miniprotein inhibitors competing with ACE2 to bind the RBD [47. Two design these molecules, two strategies were used, one exploiting the $\alpha$ helix of ACE2, and one providing de novo binders. After several rounds of optimization and testing (binding affinity measurements, solubility tests, thermostability), two designs termed LCB1 (56 amino acids) and LCB3 (64 amino acids) were selected, both binding the RBD with $K_{d}$ below $1 \mathrm{nM}$. The bound structures of LCB1 and LCB2 in complex with the RBD were also determined by cryo-EM. We use these structures to compare at a glance the interfaces (SARS-Cov-2-RBD, ACE2), (SARS-Cov-2-RBD, VHH72), (SARS-Cov-2-RBD, CR3022), (SARSCov-2-RBD, LCB1), and (SARS-Cov-2-RBD, LCB2). Remarkably, the MISA involving LCB1 and LCB3 are those which are most similar to those of ACE2 (jupyter notebook). MISA/BSA also shows a noticeable feature of the structures. Taking the example of LCB1 in complex with the RBD, the interface present in the first structure (PDBid: 7jzu) has BSA of $935.23 \AA^{2}$, while the three interfaces present in the second structure (PDBid: 7jzl) respectively have BSA of $371 \AA^{2}$ (chains A and E, RBD down), $181 \AA^{2}$ (chains C and G, RBD up), and $198 \AA^{2}$ (chains B and F, RBD up). These discrepancies owe to the much better resolution of side chains in PDBid 7jzu (jupyter notebook; Fig. 96 ).

Altogether, these illustrations stress again the systematic character of MISAs and their ability to compare at a glance coarse grain models of interfaces.

\section{Outlook}

The study of biomolecular interactions benefits from experimental and theoretical methods. On the experimental side, structure determination methods provide atomic coordinates for (ensembles) of structures, 
while thermodynamic/kinetic studies ambition to measure binding affinities and the underlying kinetic properties. Such experiments are often conducted in conjunction with directed mutagenesis, to unveil the specific role of amino acids. On the theoretical side, structure, thermodynamics and kinetics are studied using two classes of methods, namely interaction based methods, and learning based methods. As the name suggest, interaction based methods rely on covalent and non covalent potential energies models and associated force fields. Example such interactions are salt bridges, hydrogen bonds, $\pi$ stacking, etc. Such models generally have a limited spatial and time resolution, which imposes simulation methods to compute observables by means of averages over ensembles. Unfortunately, properties on long time scales are generally out of reach, so that qualitative descriptors of interaction patterns are resorted to. On the other hand, learning based method typically mix geometry based models with biochemical annotations, so as to design proxys for the key determinants of affinity and specificity. Such descriptors are known to be robust, and under suitable hypothesis, have proven good enough to estimate reliably binding affinities.

As illustrated by the example of the RBD of coronaviruses, most studies performed recently tend to promote specific interactions, usually backed up experimentally. This strategy is not entirely satisfactory, and one may actually quote [1] on the interaction (SARS-Cov-1-RBD, ACE2): "The residues singled (authors' note: the 14 a.a.) out for description in the preceding paragraphs are not, of course, the only ones critical for the tight complementarity of the SARS-CoV RBD and human (or palm civet) ACE2.". To promote a more comprehensive approach, we introduce MISAs, namely alignments between interface strings defined from geometric models, and coding biological / biophysical properties of interest. While Voronoi interface models are admittedly less precise than interaction based models, they are exhaustive/systematic, parameter free, and can be pulled back onto sequences. The encoding of interactions obtained, called interface strings, provides a concise way to capture global and local properties of complexes involving related proteins at a glance, as illustrated while analysis the RBD of coronaviruses.

We anticipate that the encoding of interfaces obtained will become a standard way to concisely represent interactions for families of homologous molecules, helping to understand protein interactions, identify critical residues, and therefore design therapeutics. 


\section{Artwork}

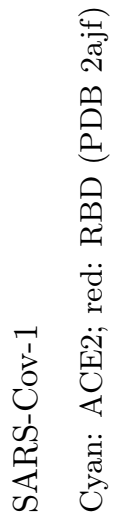

(A)

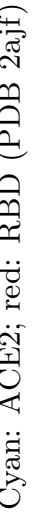

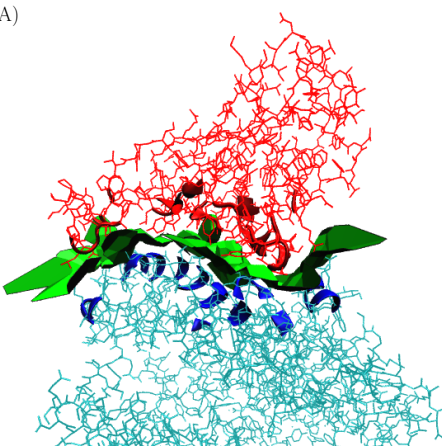

(A)

(A)

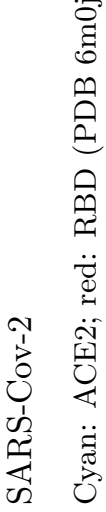

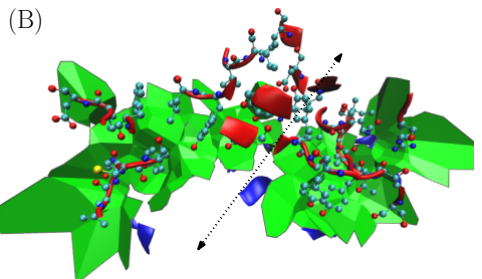

(C)

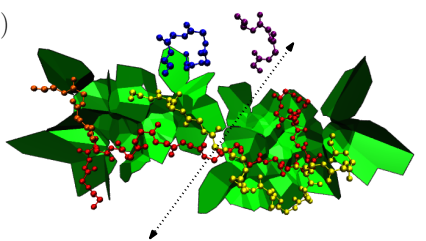

(B)

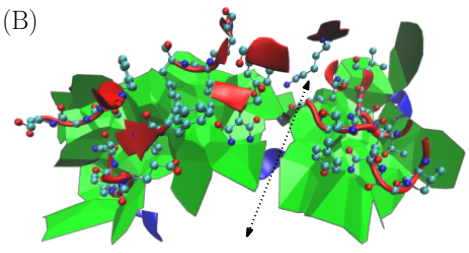

(C)

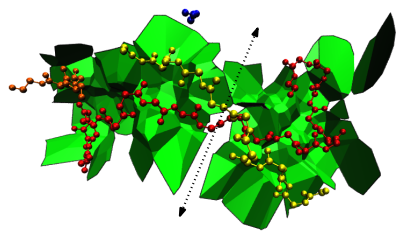

Figure 1: SARS-Cov-1 and SARS-Cov-2: Voronoi interfaces of the RBD in complex with ACE2. (Panel A) Side view of the Voronoi interface, with contributing a.a. as new ribbons. Note in particular the many disconnected contributions of SSE of the RBD to the interface. (Panel B) Top view of the interface, with interface a.a. as ribbons, and their side chains in CPK mode. (Panel C) The four or five ranges of consecutive a.a. identified by MISA, with backbone in CPK mode, on top of the Voronoi interface. SARS-Cov-1: 390-396 (violet), 404-408 (blue), 426-443 (yellow), 460-463 (orange), 470-492 (red); SARS-Cov-2: 417-421 (blue), 439-456 (yellow), 473-477 (orange), 484-506 (red) 
MISA SSE for MISA-ID SARS-COV-1-RBD_0

3-turn helix - 4-turn helix : 5-turn helix - Isolated beta-bridge residue - Extended strand
Bend hel Hydrogen bonded turn :

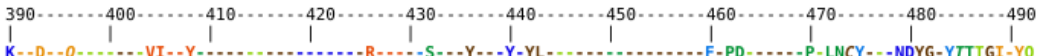

bound-2ajf-E, res :2.9 A, 29 interf res

bound-2ajf-F, res :2.9 $\AA$, 29 interf res

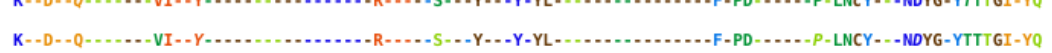

unbound-closed-5 $558-\mathrm{A}$, res : $: 3.2 \mathrm{~A}, \theta$ interf res

unbound-closed-6crz-C, res : $: 3.3 \mathrm{~A}, \theta$ interf res

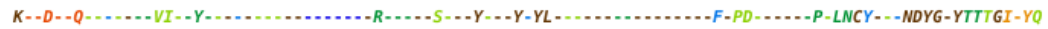

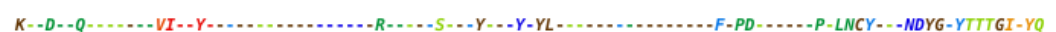

MISA SSE for MISA-ID SARS-COV-2-RBD_o

3-turn helix - 4-turn helix : 5 -turn helix - Isolated beta-bridge residue - Extended strand
Bend . Hydrogen bonded turn : other . Missing Residue

Residue Index

bound-6moj-E, res :2.45 A, 27 interf res

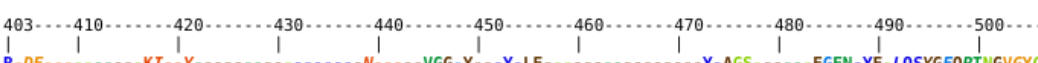

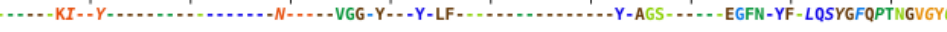

unbound-closed-6vxx-A, res :2.8 A, $\theta$ interf res

unbound-closed-6uyb- $A$, res $: 3.2 \AA, \theta$ interf res

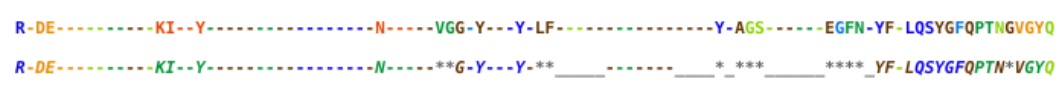

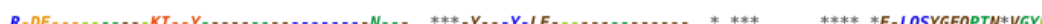

Figure 2: $\quad$ MISA/SSE for structures of Fig. 1. 


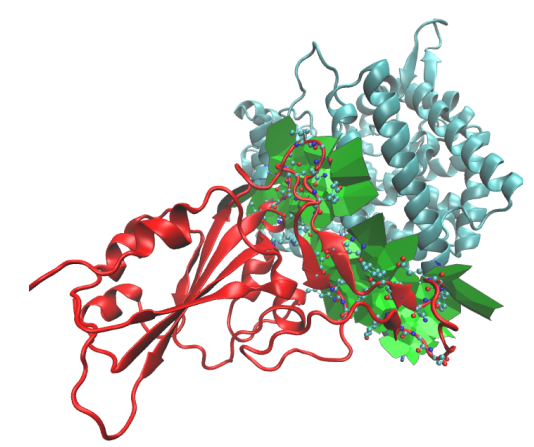

(A) SARS-Cov-2-RBD (red) - ACE2 (cyan) (PDB 6m0j)

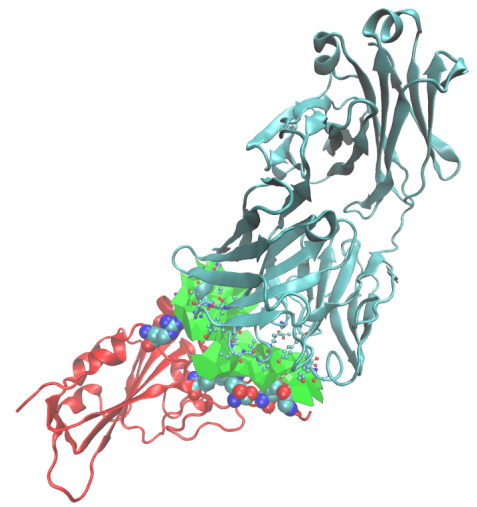

(C) SARS-Cov-2-RBD (red) - P2B-2F6 (cyan) (PDB 7bwj)

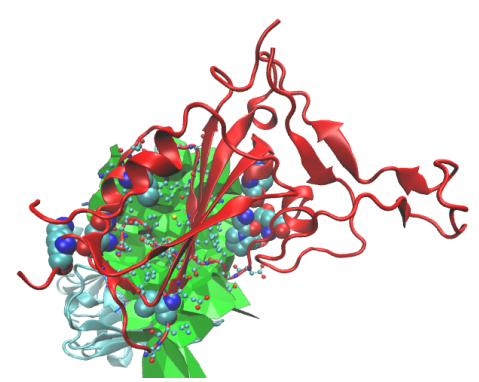

(B) SARS-Cov-2-RBD (red) - CR3022 (cyan) (PDB 6yla)

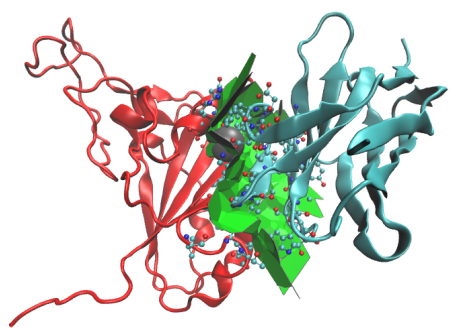

(D) SARS-Cov-1-RBD (red) - VHH-72 (cyan) (PDB 6waq)

Figure 3: SARS-Cov-2 and SARS-Cov-1: RBD bound to the various IG / VHH: Voronoi interfaces. (A) Complex with ACE2 presented for reference (A). (B) Complex from [28]. Residues in VdW mode are identified by the Voronoi interface model but are not listed in [28. (C) Complex from [36]. Residues in VdW mode are identified by the Voronoi interface model but are not listed in [36]. 


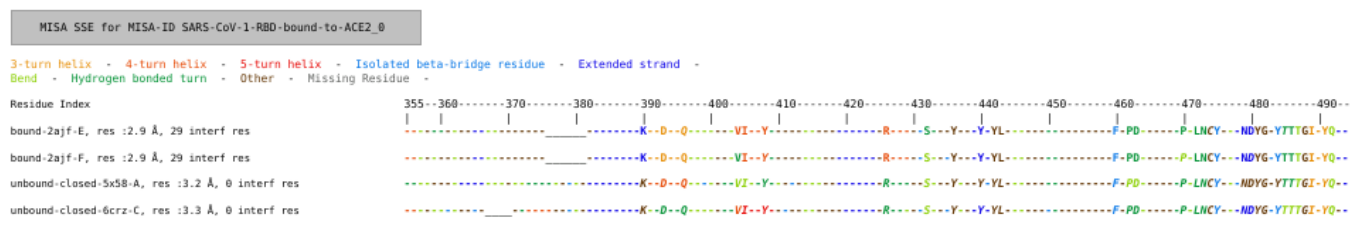

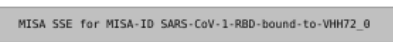

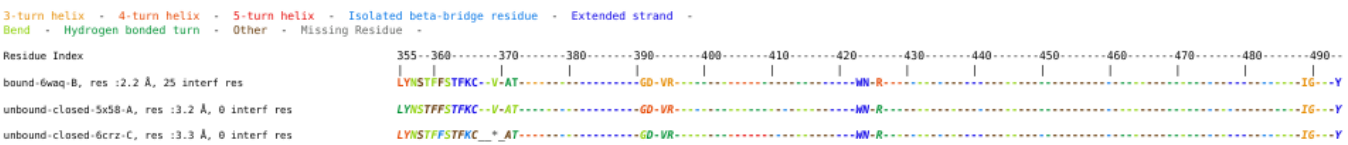

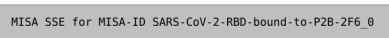

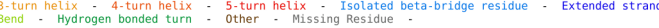

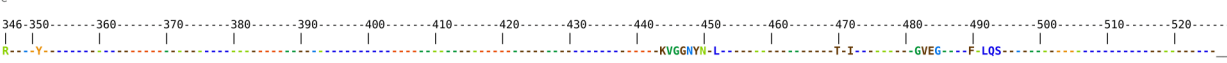

bound-7buj-E, res :2.25 A, 20 interf res

anbound-closed-6ux-A, res : :2.8 A, o interf r res

(n)

Figure 4: MISA/SSE for structures of Fig. 3. MISA of ACE2 not shown. 
Acknowledgment. T. Dreyfus is acknowledged for insightful comments. This work has been supported by the French government, through the 3IA Cote d'Azur Investments in the Future project managed by the National Research Agency (ANR) with the reference number ANR-19-P3IA-0002.

\section{References}

[1] J. Janin, R. P. Bahadur, and P. Chakrabarti. Protein-protein interaction and quaternary structure. Quarterly reviews of biophysics, 41(2):133-180, 2008.

[2] John Kuriyan, Boyana Konforti, and David Wemmer. The molecules of life: Physical and chemical principles. Garland Science, 2012.

[3] Deepa Rajamani, Spencer Thiel, Sandor Vajda, and Carlos J Camacho. Anchor residues in proteinprotein interactions. Proceedings of the National Academy of Sciences, 101(31):11287-11292, 2004.

[4] A Chang Chia-en, Wei Chen, and Michael K Gilson. Ligand configurational entropy and protein binding. Proceedings of the National Academy of Sciences, 104(5):1534-1539, 2007.

[5] H-X. Zhou and M. Gilson. Theory of free energy and entropy in noncovalent binding. Chemical reviews, 109(9):4092-4107, 2009.

[6] D. E. Shaw, P. Maragakis, K. Lindorff-Larsen, S. Piana, R. O. Dror, M. P. Eastwood, J. A. Bank, J. M. Jumper, J. K. Salmon, Y. Shan, and W. Wriggers. Atomic-level characterization of the structural dynamics of proteins. Science, 330(6002):341-346, 2010.

[7] Shi Chen, Rafal P Wiewiora, Fanwang Meng, Nicolas Babault, Anqi Ma, Wenyu Yu, Kun Qian, Hao Hu, Hua Zou, Junyi Wang, Shijie Fan, Gil Blum, Fabio Pittella-Silva, Kyle A Beauchamp, Wolfram Tempel, Hualiang Jiang, Kaixian Chen, Robert J Skene, Yujun George Zheng, Peter J Brown, Jian Jin, Cheng Luo, John D Chodera, and Minkui Luo. The dynamic conformational landscape of the protein methyltransferase SETD8. eLife, 8:e45403, may 2019.

[8] A. Schmidt, H. Xu, A. Khan, T. O'Donnell, S. Khurana, L. King, J. Manischewitz, H. Golding, P. Suphaphiphat, A. Carfi, E. Settembre, P. Dormitzer, T. Kepler, R. Zhang, A. Moody, B. Haynes, H-X. Liao, D. Shaw, and S. Harrison. Preconfiguration of the antigen-binding site during affinity maturation of a broadly neutralizing influenza virus antibody. PNAS, 110(1):264-269, 2013.

[9] Javier Corzo. Time, the forgotten dimension of ligand binding teaching. Biochemistry and Molecular Biology Education, 34(6):413-416, 2006.

[10] Mattia Bernetti, Matteo Masetti, Walter Rocchia, and Andrea Cavalli. Kinetics of drug binding and residence time. Annual review of physical chemistry, 70:143-171, 2019.

[11] F. Richards. The interpretation of protein structures: total volume, group volume distributions and packing density. Journal of molecular biology, 82(1):1-14, 1974.

[12] C. Chothia and J. Janin. Principles of protein-protein recognition. Nature, 256:705-708, 1975.

[13] S. Marillet, P. Boudinot, and F. Cazals. High resolution crystal structures leverage protein binding affinity predictions. Proteins: structure, function, and bioinformatics, 1(84):9-20, 2015.

[14] F. Cazals, F. Proust, R. Bahadur, and J. Janin. Revisiting the Voronoi description of protein-protein interfaces. Protein Science, 15(9):2082-2092, 2006.

[15] Kliment Olechnovič and Česlovas Venclovas. Voronota: a fast and reliable tool for computing the vertices of the voronoi diagram of atomic balls. Journal of computational chemistry, 35(8):672-681, 2014. 
[16] Ranjit Prasad Bahadur, Pinak Chakrabarti, Francis Rodier, and Joël Janin. A dissection of specific and non-specific protein-protein interfaces. Journal of molecular biology, 336(4):943-955, 2004.

[17] S. Marillet, M-P. Lefranc, P. Boudinot, and F. Cazals. Novel structural parameters of ig-ag complexes yield a quantitative description of interaction specificity and binding affinity. Frontiers in immunology, $34(8), 2017$.

[18] J. Pevsner. Bioinformatics and functional genomics. John Wiley \& Sons, 2015.

[19] F. Cazals and T. Dreyfus. The Structural Bioinformatics Library: modeling in biomolecular science and beyond. Bioinformatics, 7(33):1-8, 2017.

[20] S. Loriot and F. Cazals. Modeling macro-molecular interfaces with Intervor. Bioinformatics, 26(7):964$965,2010$.

[21] W. Kabsch and C. Sander. Dictionary of protein secondary structure: pattern recognition of hydrogenbonded and geometrical features. Biopolymers: Original Research on Biomolecules, 22(12):2577-2637, 1983.

[22] F. Cazals, H. Kanhere, and S. Loriot. Computing the volume of union of balls: a certified algorithm. ACM Transactions on Mathematical Software, 38(1):1-20, 2011.

[23] F. Cazals. Revisiting the Voronoi description of protein-protein interfaces: Algorithms. In T. Dijkstra, E. Tsivtsivadze, E. Marchiori, and T. Heskes, editors, International Conference on Pattern Recognition in Bioinformatics, pages 419-430, Nijmegen, the Netherlands, 2010. Lecture Notes in Bioinformatics 6282 .

[24] Lei Yang, Guang Song, and Robert L Jernigan. Comparisons of experimental and computed protein anisotropic temperature factors. Proteins: Structure, Function, and Bioinformatics, 76(1):164-175, 2009.

[25] M Alejandra Tortorici and David Veesler. Structural insights into coronavirus entry. Advances in virus research, 105:93-116, 2019.

[26] S.C. Harrison. Viral membrane fusion. Virology, 479-480:498-507, 2015.

[27] Daniel Wrapp, Nianshuang Wang, Kizzmekia S Corbett, Jory A Goldsmith, Ching-Lin Hsieh, Olubukola Abiona, Barney S Graham, and Jason S McLellan. Cryo-em structure of the 2019-ncov spike in the prefusion conformation. Science, 367(6483):1260-1263, 2020.

[28] Meng Yuan, Nicholas C Wu, Xueyong Zhu, Chang-Chun D Lee, Ray TY So, Huibin Lv, Chris KP Mok, and Ian A Wilson. A highly conserved cryptic epitope in the receptor binding domains of SARS-CoV-2 and SARS-CoV. Science, 368(6491):630-633, 2020.

[29] J. Shang, Y. Wan, C. Luo, G. Ye, Q. Geng, A. Auerbach, and F. Li. Cell entry mechanisms of SARSCoV-2. PNAS, NA(NA):1-8, 2020.

[30] Markus Hoffmann, Hannah Kleine-Weber, Simon Schroeder, Nadine Krüger, Tanja Herrler, Sandra Erichsen, Tobias S Schiergens, Georg Herrler, Nai-Huei Wu, Andreas Nitsche, et al. Sars-cov-2 cell entry depends on ACE2 and TMPRSS2 and is blocked by a clinically proven protease inhibitor. Cell, 2020.

[31] Jian Shang, Gang Ye, Ke Shi, Yushun Wan, Chuming Luo, Hideki Aihara, Qibin Geng, Ashley Auerbach, and Fang Li. Structural basis of receptor recognition by SARS-CoV-2. Nature, pages 1-4, 2020.

[32] Renhong Yan, Yuanyuan Zhang, Yaning Li, Lu Xia, Yingying Guo, and Qiang Zhou. Structural basis for the recognition of SARS-CoV-2 by full-length human ACE2. Science, 367(6485):1444-1448, 2020. 
[33] Alexandra C Walls, Young-Jun Park, M Alejandra Tortorici, Abigail Wall, Andrew T McGuire, and David Veesler. Structure, function, and antigenicity of the SARS-CoV-2 spike glycoprotein. Cell, 2020.

[34] Alexandra C Walls, Xiaoli Xiong, Young-Jun Park, M Alejandra Tortorici, Joost Snijder, Joel Quispe, Elisabetta Cameroni, Robin Gopal, Mian Dai, Antonio Lanzavecchia, et al. Unexpected receptor functional mimicry elucidates activation of coronavirus fusion. Cell, 176(5):1026-1039, 2019.

[35] D. Wrapp, D. De Vlieger, K. Corbett, G. Torres, N. Wang ad W. Van Breedam, K. Loes van Schie, M Hoffmann, S. Pohlmann, B. Graham, N. Callewaert, B. Schepens, X. Slelens, and J. McLellan. Structural basis for potent neutralization of betacoronaviruses by single-domain camelid antibodies. Cell, 2020.

[36] B. Ju, Q. Zhang, J. Ge, R. Wang, J. Sun, X. Ge, J. Yu, S. Shan, B. Zhou, and S. Song. Human neutralizing antibodies elicited by sars-cov-2 infection. Nature, pages 1-8, 2020.

[37] BERNARD B. Delmas and H. Laude. Assembly of coronavirus spike protein into trimers and its role in epitope expression. Journal of virology, 64(11):5367-5375, 1990.

[38] F. Li. Structure, function, and evolution of coronavirus spike proteins. Annual review of virology, $3: 237-261,2016$.

[39] Alexandra C Walls, M Alejandra Tortorici, Joost Snijder, Xiaoli Xiong, Berend-Jan Bosch, Felix A Rey, and David Veesler. Tectonic conformational changes of a coronavirus spike glycoprotein promote membrane fusion. Proceedings of the National Academy of Sciences, 114(42):11157-11162, 2017.

[40] J. Jaimes, N. André, J. Chappie, J. Millet, and G. Whittaker. Phylogenetic analysis and structural modeling of SARS-CoV-2 spike protein reveals an evolutionary distinct and proteolytically-sensitive activation loop. Journal of molecular biology, 432:3309-3325, 2020.

[41] Fang Li, Wenhui Li, Michael Farzan, and Stephen C Harrison. Structure of SARS coronavirus spike receptor-binding domain complexed with receptor. Science, 309(5742):1864-1868, 2005.

[42] J. Janin. A minimal model of protein-protein binding affinities. Protein Science, 23(12):1813-1817, 2014.

[43] Sara Birtalan, Yingnan Zhang, Frederic A Fellouse, Lihua Shao, Gabriele Schaefer, and Sachdev S Sidhu. The intrinsic contributions of tyrosine, serine, glycine and arginine to the affinity and specificity of antibodies. Journal of molecular biology, 377(5):1518-1528, 2008.

[44] Shohei Koide and Sachdev S Sidhu. The importance of being tyrosine: lessons in molecular recognition from minimalist synthetic binding proteins. ACS chemical biology, 4(5):325-334, 2009.

[45] Philip Lijnzaad, Herman JC Berendsen, and Patrick Argos. Hydrophobic patches on the surfaces of protein structures. Proteins: Structure, Function, and Bioinformatics, 25(3):389-397, 1996.

[46] D. Corti and A. Lanzavecchia. Broadly neutralizing antiviral antibodies. Annual review of immunology, 31:705-742, 2013.

[47] L. Cao, I. Goreshnik, B. Coventry, J.B. Case, L. Miller, L. Kozodoy, R. Chen, L. Carter, A. Walls, Y-J. Park, E-M Strauch, L. Stewart, M.S. Diamond, D. Veesler, and D. Baker. De novo design of picomolar SARS-CoV-2 miniprotein inhibitors. Science, 370(6515):426-431, 2020. 
6 Supplemental: results 
MISA BSA for MISA-ID SARS-COV-1-RBD

In dark grey, residues with missing data for coloring

Buried Surface Area (BSA) (in $\left.A^{2}\right)$

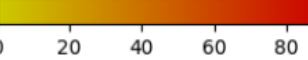

I bound-2ajf-E : total bsa $=925.41 \AA^{2} \mid$ bound $-2 a j f-F:$ : total bsa $=864.87 \AA^{2}$
Residue Index $\begin{array}{ll} & \\ \text { bound-2ajf-E, res :2.9 A, } 29 \text { interf res } & 1\end{array}$

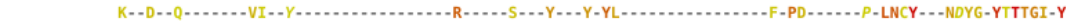

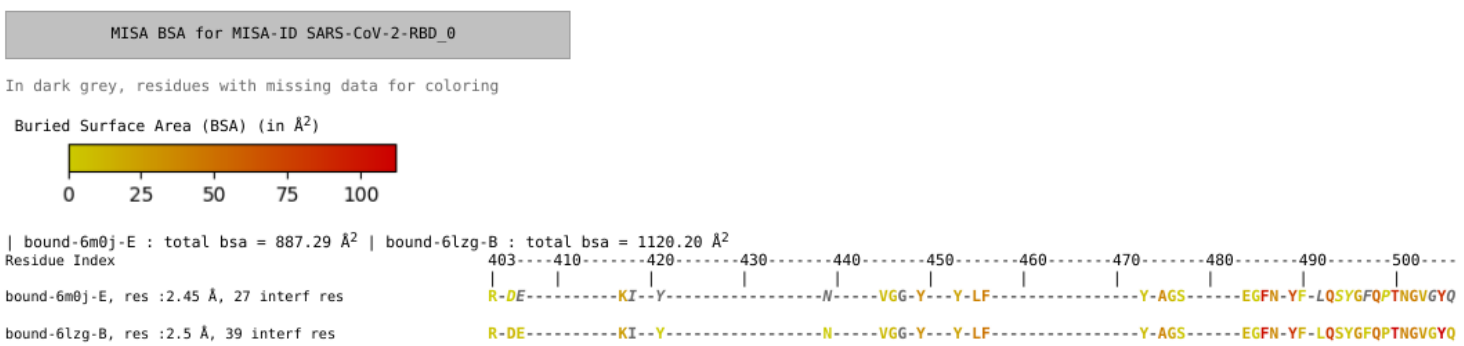

Figure S 1: MISA/BSA for Fig. 1. 
MISA Delta_ASA for MISA-ID SARS-COV-1-RBD_。

In dark grey, residues with missing data for coloring

Bound structures: In light grey residues in bound structure for which miss corresponding ASA values in the unbound structures

$$
50 \quad 100 \quad 150
$$

bound-2ajf-E, res :2.9 A, 29 interf res

bound-2ajt-F, res :2.9 A, 29 interf tes

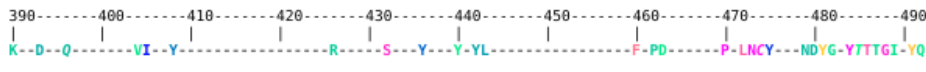

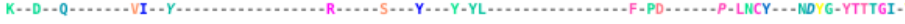

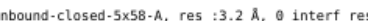

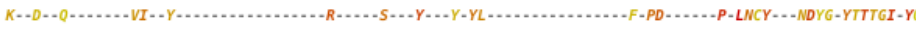

unbound-closed-6crz-C, res $: 3.3 \AA$, a interf res

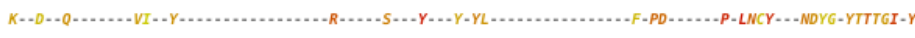

MISA Delta_ASA for MISA-ID SARS-COV-2-RBD__

In dark grey, residues with missing data for coloring

Bound structures: In light grey residues in bound structure for which miss corresponding ASA values in the unbound structures

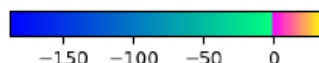

Unbound structures : Accessible Surface Area (ASA) (in $\left.\AA^{2}\right)$

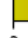

100

200

Residue Index

bound-6nej-E, res :2.45 A, 27 interf res

bound-6lzg-B, res :2.5 A, 39 interf res

unbound-closed-6vxx-A, res :2.8 $\mathrm{A}, \theta$ interf res

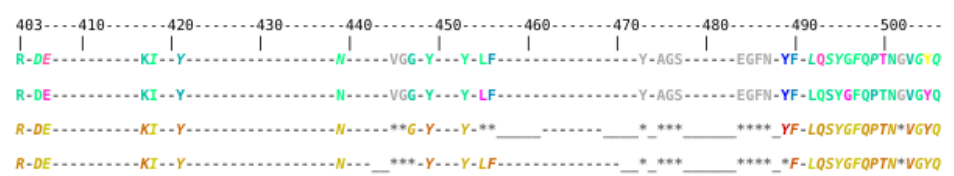

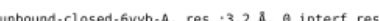

Figure S 2: MISA/ $\Delta$ ASAfor Fig. 1 . 
Hierarchical clustering for SARS-CoV-2-rbd_0_interface_with_grainmode_1_with_40_residues_ref with the average method
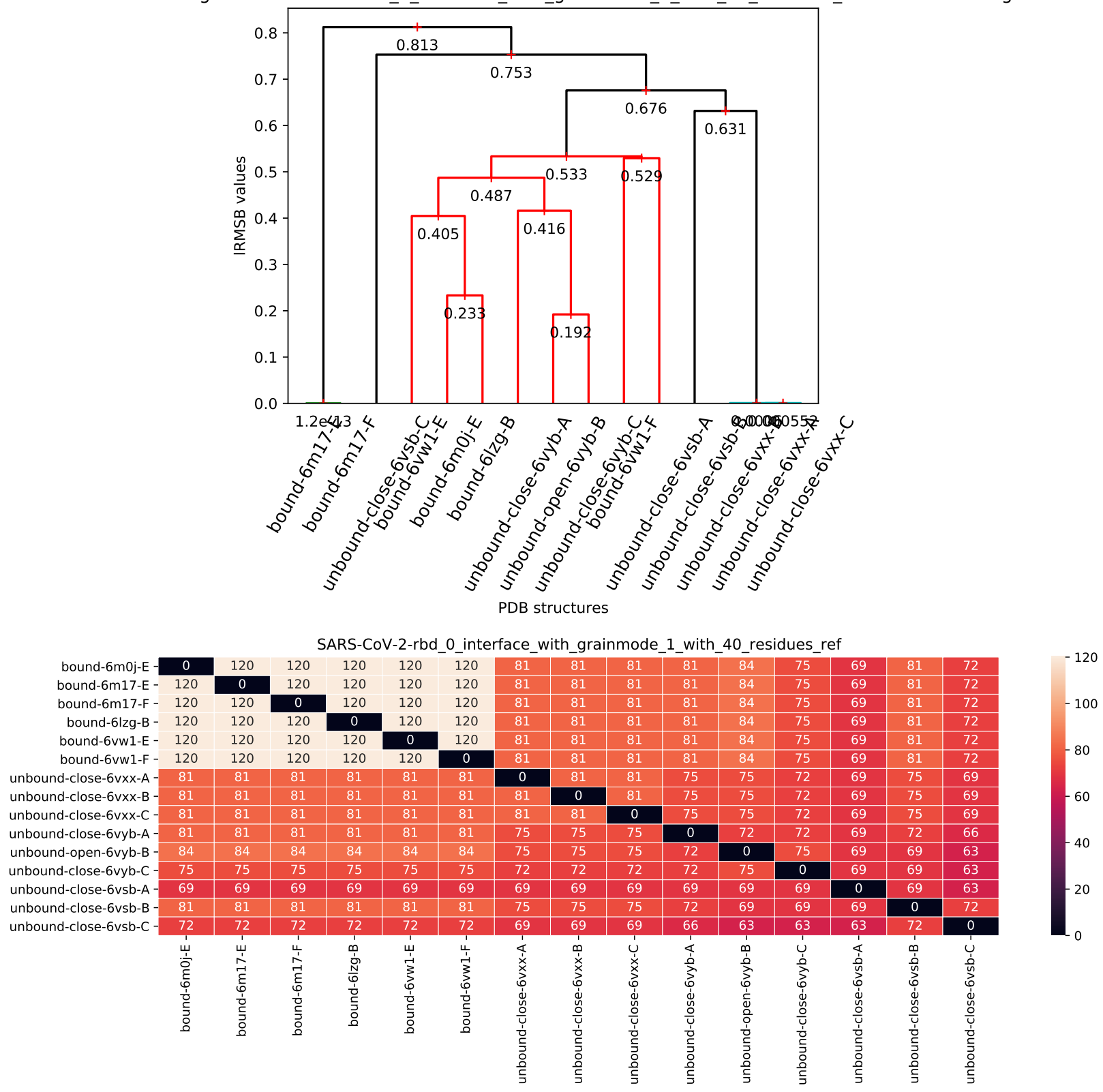

Figure S 3: Hierarchical clustering of conformation of the RBD, based on the iRMSD of backbone atoms. Each individual iRMSD is computed based on the backbone atoms common to two structures (selected loops may be missing in a given structure). (A) Dendrogram (B) Num. backbone atoms in an individual comparison. 

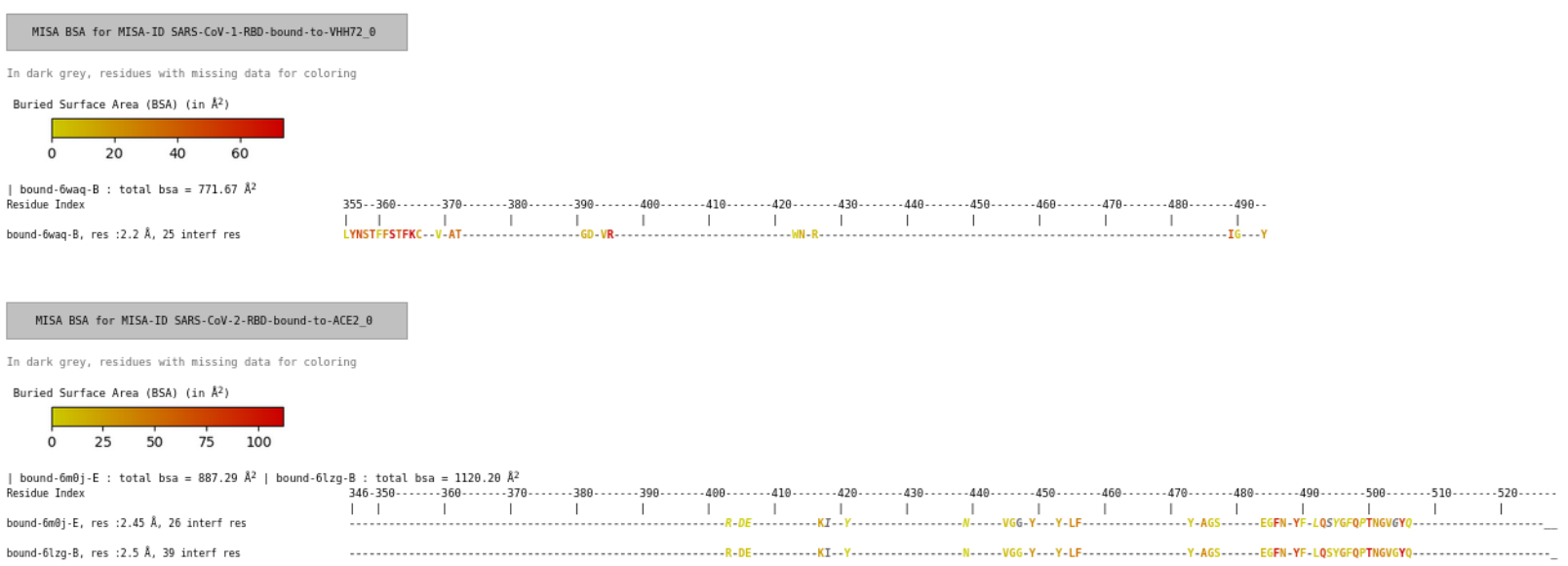

bound-627g-B, res :2.5 A, 39 interf res

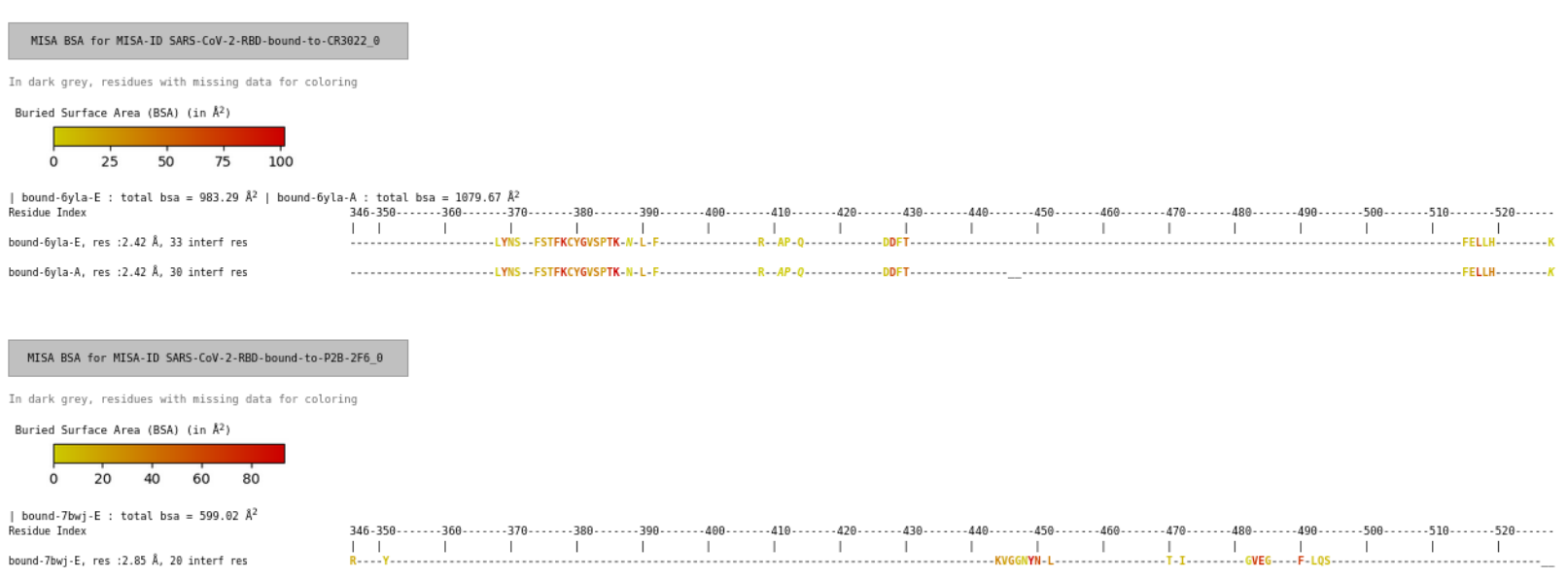

Figure S 4: MISA/BSA for Fig. 3. 


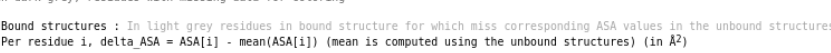

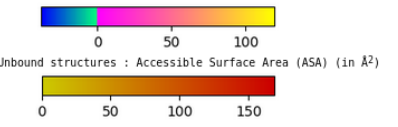

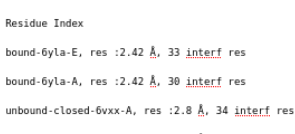

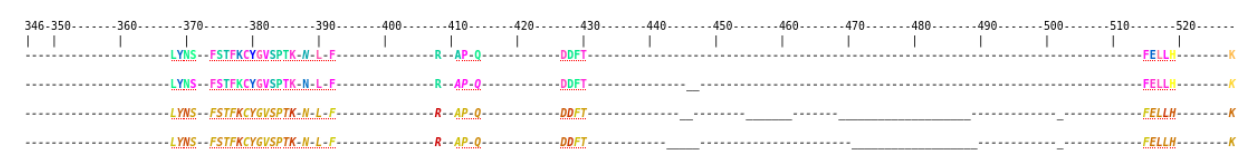

unbound-closed-6yyb-A, res :3.2 A, 34 intert res

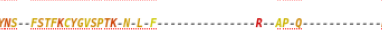

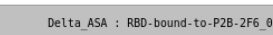

In dark grey, residues with nissing data for coloring

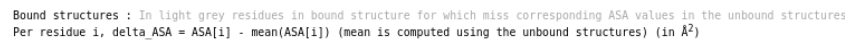

\begin{tabular}{|llllll}
-60 & -40 & -20 & 0 & 20 \\
-70 & 0
\end{tabular}

Unbound structures : Accessible Surface Area (ASA) (in A $A^{2}$ )

50 $\quad 100 \quad 150$

Residue Index

bound-7brj-E, res :2.85 A., 20 intert res

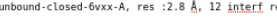

unbound-closed-6yyb-A, res : $3.2 \mathrm{~A}$, 11 intert res

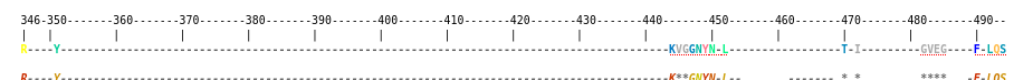

Delta ASA : R80-bound-to. -VHA-72.

ndark grey, residues with missing data for coloring

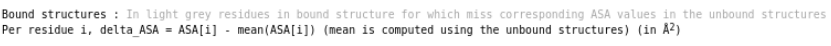

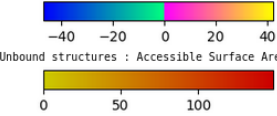

Residue Index

100

bound-6.rana-B, res :2.2. A. 25 intert res

unbound-closed-5.558-A, res : 3.2 .2 A. 25 intert res

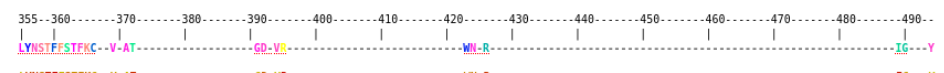

unbound-closed-6erz-c., res :3.3 \&. 24 intert res

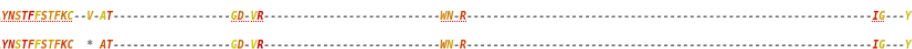

Figure S 5: MISA/ $\Delta$ ASAfor Fig. 3. 


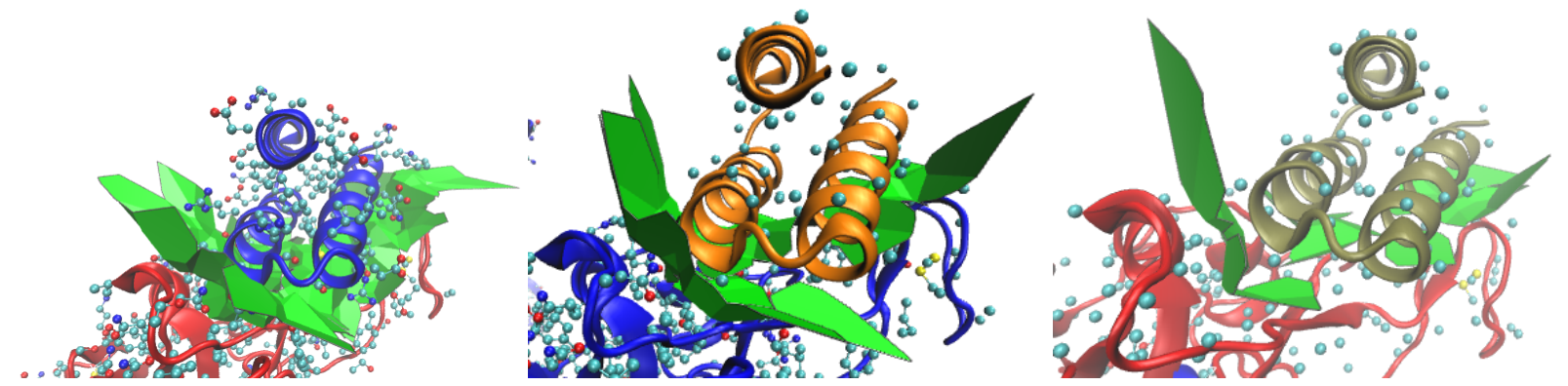

Figure S 6: Interfaces between LCB1 and the RBD in two different structures [47]. New cartoons: molecules; CPK representations: side chains; green polygons: Voronoi interface between the two partners. Left: PDBid 7jzu, chains $\mathrm{A}$ and $\mathrm{B}, \mathrm{BSA}=935.23 \AA^{2}$; Middle: PDBid 7jzl, chains $\mathrm{A}$ and $\mathrm{E}, \mathrm{BSA}=371$ $\AA^{2}$; Right: PDBid 7jzl, chains B and F, BSA $=198 \AA^{2}$. Discrepancies in the BSA owe to a much better resolution of side chains in PDBid 7jzu. 


\section{Supplemental: formal specification of the method}

We formalize the notions introduced in the main text.

Voronoi interface restricted to a chain. We first process the Voronoi interface of a complex $C_{i}$ and collect all the interface amino-acids for a chain instance $S_{i j}=C_{i}\left[A_{j}\right]$ :

Definition. 5 (Half interface) Consider the Voronoi interface model for the partners $\left\{A_{j}\right\}$ and $\left\{B_{j}\right\}$ in structure $C_{i}$. The restriction of the Voronoi interface to chain $S_{i j}$, or half interface for short, is the set of a.a. of this chain contributing at least one atom at the interface. This set is denoted $I_{i j}^{\text {Vor. }}$. The operator $I_{i j}^{\text {Vor. }}[k]$ returns the one letter code of the $k$-th a.a. of $S_{i j}$ if this a.a. is at the interface, and $\emptyset$ otherwise.

Considering all chains associated with a given MISA id yields a set of a.a. at a given position, amidst which we seek the most frequent one:

Definition. 6 The interface pool of chain $A_{j}$ at position $k$ is defined as $P_{j, k}=\cup_{i} I_{i j}^{\text {Vor. }}[k]$, where index $i$ ranges over all complexes. The consensus a.a. at position $k$ is the a.a. most frequent in the pool $P_{j, k}$, with ties broken using the lexicographic on the one letter code of the amino-acids.

Interface strings. To encode properties of a particular chain instance $S_{i j}$ in a structure (bound or unbound), several parameters must be taken into account: the fact that this position is involved in interfaces or not, the potential absence of this position in selected crystal structures, and also the variability observed at that position (Def. 6). These pieces of information are precisely summarized in the so-called interface string, which is a string defined over the alphabet using the one letter code of a.a. plus the three symbols $\{-*\}$ :

Definition. 7 (Interface string) The interface string $\mathcal{I}_{i j}$ of chain $A_{j}$ in structure $C_{i}$ or $U_{i}$ ) is the string with one character per amino-acid, defined as follows:

- (Case 1) k-th a.a. never at the interface i.e. $P_{j, k}=\emptyset:$ _ if this a.a. is absent from the crystal structure, and - otherwise.

- (Case 2) $k$-th a.a. sometimes at the interface, but not in $S_{i j}$ i.e. $P_{j, k} \neq \emptyset$ and $I_{i j}^{\text {Vor. }}[k]=\emptyset$ : italicized capital one letter code $X$ for the consensus a.a., italicized lowercase one letter code $x$ otherwise.

- (Case 3) $k$-th a.a. at the interface i.e. $I_{i j}^{\text {Vor. }}[k] \neq \emptyset$ :

- * if a.a. is absent from the crystal;

- capital one letter code $\mathrm{X}$ for the consensus a.a., lowercase one letter code $\mathrm{x}$ otherwise.

Note that for a chain in an unbound structure, Case 3 is irrelevant since there is no interface.

Collecting all such strings for a given MISA id yields Def. 4 . 


\section{Implementation details}

In the following, we provide the implementation details, using in particular the notations of Section 7 for the operators.

\subsection{Overview}

sbl-misa.py is the main script of the package: it creates the MISAs, colors them and performs a structural comparison between the different chains of the same MISA. It uses different modules that we present here, through their main classes.

From its output, different complementary scripts can be used to further the analysis and/or parameterize the display of the MISAs. This section follows the organization of sbl-misa.py, and the different steps of the script refer to their numbering in Fig 7 .

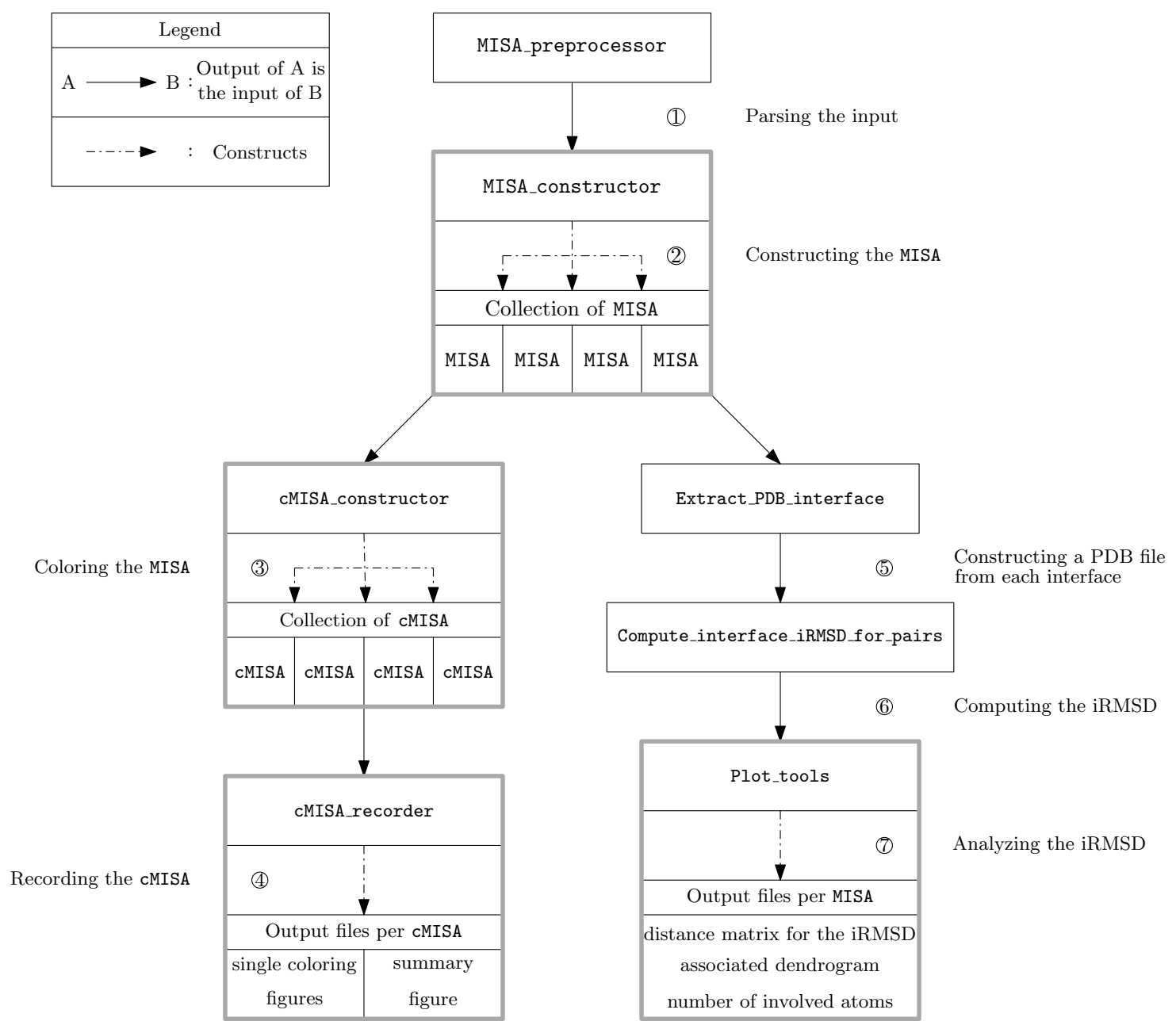

Figure S 7: Overview of sbl-misa.py 


\subsection{Computing the MISA : script sbl-misa.py, module Multiple_interface_string_alignment.py}

Overview Multiple_interface_string_alignment.py creates the MISA from a specification file and the input data described above. First, it gathers the homologous structures together, by giving them the same MISA id, and further computes from this set of chains several operators $\left(S_{i j}[k], I_{i j}^{\text {Vor. }}[k], P_{j, k}\right)$. These operators, as presented above, depend on two indices: $i$ which designates the complex index and $j$ which designates the MISA id. In the code, these operators are implemented at constant $j$, i.e. there is one instance of each operator by MISA id. These operators are used to compute the consensus interface, and from there to create an istring for each chain. The simultaneous display of all the istring corresponding to the same MISA id is a MISA.

\section{Main steps and associated classes}

\section{- Step 1. : Parsing the input}

Main class : class MISA_preprocessor

Parse the specification file and gather the structural data

sbl-misa.py starts by reading the input files, and possibly runs sbl-intervor-ABW-atomic.exe and/or sbl-vorlume-pdb.exe. (The script controls that every structure has a coherent number of chains among the different complex specifications, and if it is not the case, it runs only on the complete structures). It parses the input specification file, and infers the MISA id of each chain.

\section{- Step 2. : Constructing the MISA}

Main class : classes MISA_constructor, MISA

Initialize the MISA by gathering the chains with the same MISA id and aligning them

In the following, we assume a mapping between the original indexing of the residues in each chain, and the renumbering in the MSA. For homologous chains, this mapping may be computed ClustalOmega. The construction of the MISA involves three steps:

- Initializating the MISA, class MISA_constructor : All the chains sharing the same MISA id are gathered into an instance of the class MISA, which itself contains one instance of the Chain instance operator $S_{i j}[k]$, one instance of the Interface_oneside operator $I_{i j}^{\text {Vor. }}[k]$ and one instance of the Interface_string operator $\mathcal{I}_{i j}[k]$ (cf. Fig 22). The classes implementing these three operators have the corresponding names (Chain_instances, Interface_oneside, Interface_strings).

- Constructing the consensus interface, class Interface_pool : The consensus interface is computed for each MISA id from the set of the bound chains, by taking the most frequent residue at each position.

- Constructing the i-string, class MISA : for each MISA, we compute its instance of $\mathcal{I}_{i j}[k]$, by comparing the residues with the consensus interface and marking them accordingly. Only the residues which are part of a windows, if a windows was provided, are displayed.

Remark 3 Practically, each residue is represented as a triple (aa-or-hetero-code, resid, insertion code), which makes it possible to handle insertion codes. This representation is used both for the keys and values of the aforementioned mapping. The corresponding class is Residue_mapping.

\subsection{Coloring the MISA : script sbl-misa.py, modules Colored_MISA.py, MISA_ColorEngines.py}

Overview sbl-misa.py produces four colorings for each MISA, one for each of the following quantities (designated as coloring values):

- the secondary structure $(S S E)$ 


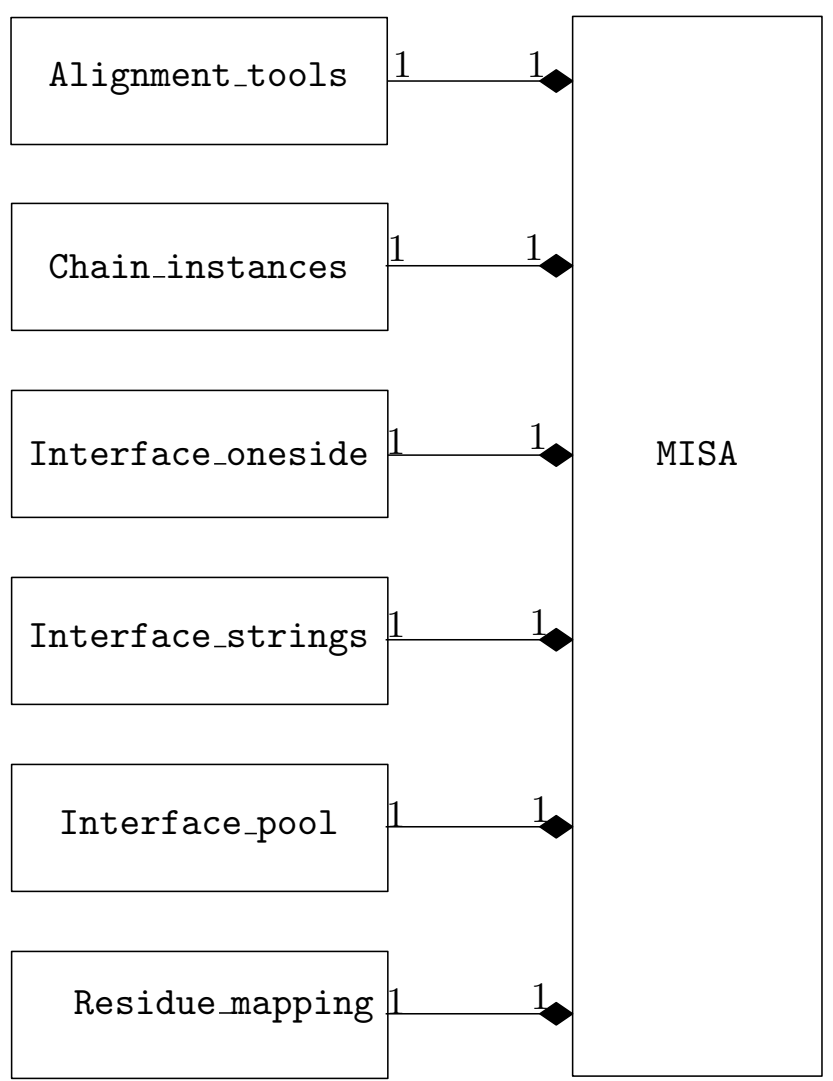

Figure S 8: Composition of the MISA class. 


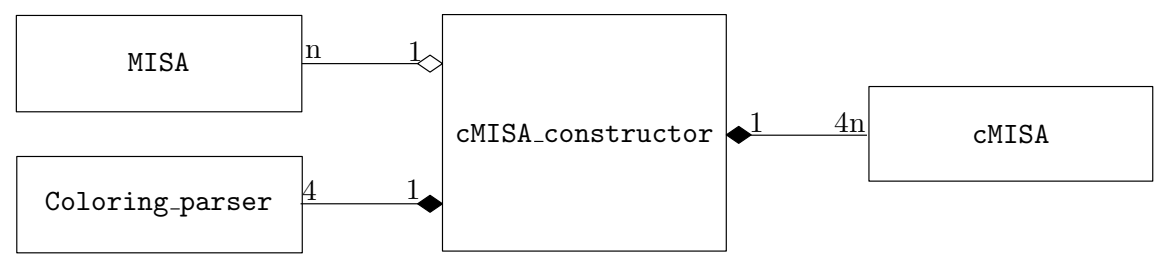

Figure S 9: Composition of the CMISA_constructor class

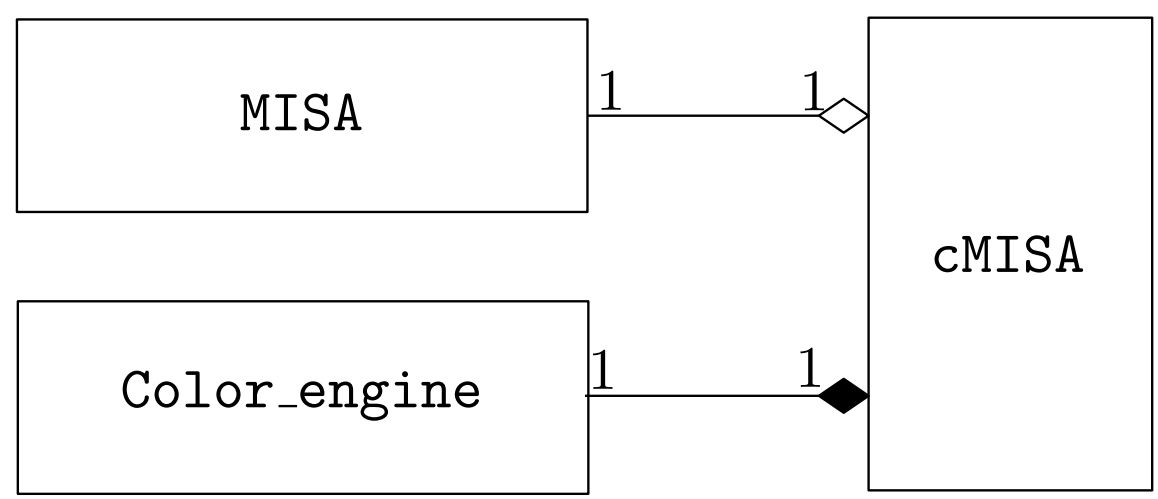

Figure S 10: Composition of the cMISA class

- the buried surface area $(B S A)$

- the difference between the accessible surface area $(A S A)$ when changing from unbound to bound structure $\left(\Delta_{-} A S A\right)$

- the B-factor

\section{Main steps and associated classes}

- Step 3. : Coloring the MISA

Main class : classes CMISA_constructor, coloring_parser, color_engine

Collect the coloring values and attribute the coloring to each chain of the MISA

- Collect of the coloring values, classes SSE_coloring_parser, BSA_coloring_parser, Delta_ASA_coloring_parser, B_factor_coloring_parser : The coloring values for all the chains are collected by the four coloring_parsers.

- Creation of the Colored MISA (cMISA), class CMISA_constructor: For each MISA, the cMISA_constructor initializes one color_engine per coloring_parser, and creates one instance of the class cMISA per pair of MISA - color_engine. (Thus we have \#cMISA = \#MISA X \#color_engine, with \#color_engine =4) (cf. Fig 3)

- Application of the coloring, classes SSE_color_engine, BSA_color_engine, Delta_ASA_color_engine, B_factor_color_engine : In each cMISA (cf. Fig 3), the color_engine assigns to each of the residues of the MISA its coloring value, and translates it by the corresponding color, by updating its Interface_string.

- Step 4. : Recording the cMISA

Main class : class CMISA_recorder 
Record the colored MISA into an HTML file presenting simultaneously the four colorings

The cMISA_recorder creates the output files, by combining the Interface_string of each cMISA with the coloring legend provided by the color_engine of the cMISA. For each cMISA, the chains with the same output are grouped together to lighten the display. A .html file displaying simultaneously all the cMISA sharing the same MISA id is created in the odir/MISA directory. A sub-directory odir/MISA/single-coloring-figures is created, where each of the cMISA is stored individually, in both .pdf and .html format.

\subsubsection{Coloring SSE}

As explained in Section Colored MISAs, we color aa in interface strings to show changes in the $\mathrm{H}$ bonding network. To this end, we use DSSP [21] and https://swift.cmbi.umcn.nl/gv/dssp/. The corresponding color code reads as follows:

- 3-turn helix : light orange

- 4-turn helix : deep orange

- 5-turn helix : red

- Isolated beta-bridge residue : light blue

- Extended strand : deep blue

- Bend : light green

- Hydrogen bonded turn : deep green

- Other : brown

- Missing Residue : grey

\subsubsection{Coloring BSA and $\Delta_{-} A S A$}

The BSA is computed using the intervor "-bsa" option. For this coloring mode, only bound structures are displayed, as it would make no sense to do so for unbound structures, which necessarily have no BSA.

The ASA is computed using sbl-vorlume-pdb.exe, and the $\Delta_{-} A S A$ is deduced from it, as explained in the main text. We display thus the $\Delta_{-} A S A$ for the bound structures, and the ASA for the unbound structures. (If only bound structures were provided, the $\Delta_{-} A S A$ won't be computable, and the sequence will be displayed in grey).

We use one color map for the BSA coloring, and two for the $\Delta_{-} A S A$ coloring.

\subsubsection{Coloring B-factor}

The B-factor is extracted from the PDB file (and possibly computed from ANISOU, see main text, if only ANISOU is available). We use one color map for the B-factor coloring.

\subsection{Script sbl-misa-mix.py}

Overview The sbl-misa-mix.py script allows, based on the individual colored MISA produced by sbl-misa.py, to display together several colored MISA for an easier comparison.

\section{Main steps}

- Parse the mix_ifile

- Parse the several .html files containing the MISA id / colorings of interest

- Combine them into a mixed fig 


\subsection{Script sbl-misa-bsa.py}

Overview sbl-misa-bsa.py provides access to the numerical values of the buried surface area (BSA) at the residue level, by parsing the .xml file produced by sbl-intervor-ABW-atomic.exe that contains the BSA data.

\section{Main steps}

- Parse the spec_file

- Parse the xml file

- Extract the BSA from the appropriate residues

\subsection{Script sbl-misa-diff.py}

Overview sbl-misa-diff.py allows to compare the interface of two homologous chains, by comparing the list of their residues at the interface.

\section{Main steps}

- Parse the spec_file

- Gather the data for the different interfaces, either in parsing an interface-file, or the MISA output.

- Compare the two interfaces

\subsection{Structural comparison, script sbl-misa.py, module iRMSD.py}

Overview sbl-misa.py finally also computes the i-RMSD for each pair of chains within each MISA, to compare the geometry of the different homologous chains.

\section{Main steps and associated classes}

\section{Step 5. : Construction of the PDB interface files}

Main class : class Extract_PDB_interface

Store the interface residues shared by each pair of chain into PDB files

Creates for each pair of chain of each MISA two new .pdb files (one per chain), which only contains the interface residues shared by both chains. The selection of the residues at the interface is done thanks to the helper class ResSelector.

\section{- Step 6. : Computation of the i-RMSD for each pair of chain}

Main class : class Compute_interface_iRMSD_for_pairs

Compute the iRMSD for each pair of PDB interface files

Compute the iRMSD for each pair of chains by calling the script sbl-lrmsd-for-pdb-pair.exe for each pair of .pdb files precedently created. The l-RMSD is computed for three different 'grainmodes' (0: only the C-alpha, 1: the backbone, 2: all the atoms)

\section{- Step 7. : Analysis of the i-RMSD}

Main class : class Plot_tools

Cluster the chains according to the iRMSD and record statistics on the iRMSD

Once we have the i-RMSD for each MISA, we derive three figures from it :

- The first is a dendrogram showing the hierarchical clustering of the i-RMSD.

- The second is a distance matrix showing the value of the i-RMSD for each pair of chains. 
- The third is a matrix showing the number of atoms involved in the calculation of i-RMSD for each pair of chains.

As the i-RMSD is computed for each of the three grainmodes above, these three figures are produced for each of them, and the grainmode is indicated in their title. 